\title{
An Enhanced Coding Algorithm for Efficient Video Coding
}

\author{
V. R. Prakash ${ }^{*}$, Saran \\ Electronics and Communication Engineering, St. Michael engineering college, Madurai, India \\ *Corresponding author: prakashkrishna53@gmail.com
}

\begin{abstract}
How to cite this paper: V. R. Prakash, Saran (2019) An Enhanced Coding Algorithm for Efficient Video Coding. Journal of the Institute of Electronics and Computer, 1, 18-28.

https://doi.org/10.33969/JIEC.2019.11004.

Received: November 25, 2019

Accepted: December 20, 2019

Published: December 24, 2019

Copyright $($ C 2019 by author(s) and Institute of Electronics and Computer. This work is licensed under the Creative Commons Attribution International License (CC BY 4.0).

http://creativecommons.org/licenses/by/4.0/
\end{abstract}

\begin{abstract}
With the advancement in modern video processing technologies, the requirement of an efficient coding algorithm for compressing the video data in a huge surveillance system is necessary. The video data rate in a surveillance system plays a crucial role in determining the performance of the compression algorithm. In this paper, a novel compression algorithm for effectively compressing the video inputs from the surveillance systems is presented. The traditional methods fail to eliminate the redundancies in the video input, and can't meet the current requirement standards in the modern technologies. This results in the increased storage requirement for input video and also makes it time-consuming in processing the video input in real time. In order to overcome the above issues, the proposed algorithm made sufficient modification in the traditional run length coding algorithm by encoding the frames and removing the redundancies using the texture information similarity in the surveillance video, thereby achieved a better compression rate of $50 \%$ for a huge dataset of surveillance videos when compared to existing methodologies.
\end{abstract}

\section{Keywords}

Run Length Coding; Global Redundancies; Compression Ratio; Texture Feature; Similarity Measurement.

\section{Introduction}

In last few decades, the role of real-time surveillance systems plays a major role in most of the security applications and remote monitoring systems etc. In most of the applications, the quality of output from the surveillance system becomes crucial and hence cameras having huge pixel quality are used. This results in the requirement of huge storage resources and also increases the system complexity to process the video data in real-time applications. In order to overcome the above issue, a lot of 
researches have been carried out to compress the video data without sacrificing the performance of the system. Jing Xiao et al. [1] observed that the presence of large of amount of redundancies due to static objects or background information's in the big surveillance video data (BSVD) has to be removed in order to provide better compression ratio. Amritha et al. [2] use the approach of Transform domain Wyner - Ziv (TDWZ) video coding techniques which utilizes Set Partitioning In Hierarchical Tree (SPIHT) instead of traditional discrete cosine transform in distributed video coding in order to have a fast and efficient technique for compression. Recently Markov process is utilized for transformation or intra prediction performance but not in both the techniques. Kamisli, F [3] incorporates Markov process on both 2D transform and the interframe coding which results in improved coding gains and produces less blocking effects at low bitrates. Minsheng et al. [4] proposed a redundancy mechanism of Global Object Redundancy (GOR) which is obtained by the movement of the vehicles. It is based on the features and the model and establishes high-quality compression since only few vehicle objects individual semantic and feature parametric can be transferred and coded. An Adaptive Golomb-Rice coding scheme based context modeling is implemented in conjunction with complexity reduction and an adaptive arithmetic coder is used in [5]. Highly dynamic range (HDR) compression can be done by two methods, namely tone mapping with backward compatibility and tone mapping without backward compatibility as in [6]. It has been analyzed in two manners, joint compression and encryption algorithm and the other as compression independent encryption algorithm [7]. The compressibility constrained sparse representation (CCSR) approach is proposed in the paper [8] for the compression of images at low bit-rate. The compression technique is proposed along with the matching pursuit (MP) algorithms by using texture reinforcements of the dictionary. The background-modelling-based adaptive prediction (BMAP) method is implemented for the purposes of prediction of background. The background difference prediction (BDP) predicts the data of the domain currently obtained and it improves the efficiency by the references of background [9]. In paper [10] the compression video analysis is based on the block SVD Algorithm. It reduces the representing of bits of video frames with the reduction of space for storage and the cost for the transmission during the management of good quality. It provides better performances of PSNR at the same bit rate with the complexity of time reduction. The low-rank and sparse decomposition (LRSD) is used to compress the video with the component of low rank by representing the object, components and the background. The temporal redundancy is removed by the dependency of a linear process of background frames [11]. In this paper, a novel video compression 
algorithm is presented with improved compression rate and reducing the amount of storage space required. The algorithm is developed using MATLAB 8.2 and its performance is compared with traditional compression algorithms.

\section{Proposed Algorithm}

The proposed work mainly concentrates on the development of Improved Run Length Coding Scheme (IRLC) for the reduction of disk space required, global redundancy, and amount of time needed for processing the data and the compression rate. The stages involved in the development of Improved Run Length Coding Scheme (IRLC) are shown in Figure 1. The first stage of the proposed algorithm involves preprocessing the video data and extraction of video frames from the video input. After extracting frames, the salient features including texture, color etc., are identified. Once it is identified, those frames processed with the color space conversion can determine the individual layers. Later the features are extracted from the processed frames in order to identify the redundant information. Based on extraction method, the features are extracted and applied to the next process of redundancy removal and for estimation of the similarity measure. Then the data is encoded by the enhanced run length coding approach for the reduction of space and time. The compressed bit stream is obtained and decoding the process is implemented in the reverse process of encoding for reconstruction of video.

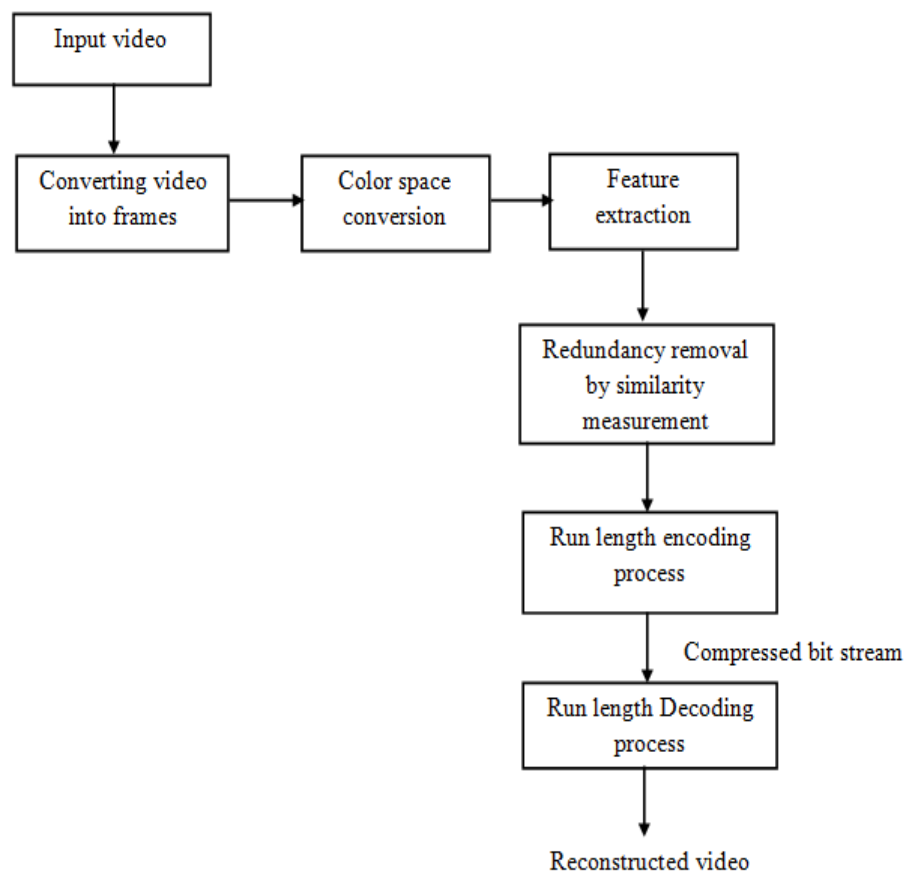

Figure 1. Workflow of Proposed Algorithm 
The processes of redundancy removal and the feature extraction are carried out as per the procedure of the proposed approach. The salient feature is used to detect the data with the key attention mechanism which enables the process of availability of the data between the neighbors and items. It describes the visual of the data with the consideration of reactive process, bottom-up and the memory dependent. It integrated the influences by splitting it as top-down and bottom-up. It enables the ability for the allocation limits of resources for the input of visual quality. The visual process of the model mechanism is determined as the visual salient detection. Then the frames are processed by applying the LAB color space.

\subsection{LAB space model}

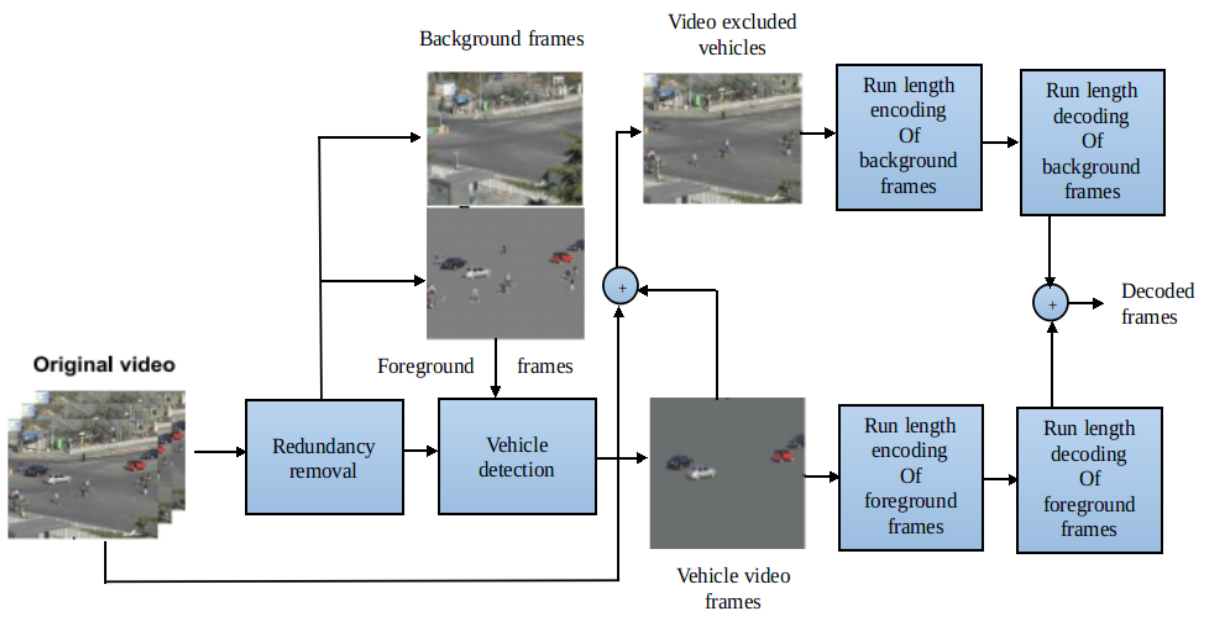

Figure 2. Block Diagram of IRLC approach

In LAB color space, the dimension and space as per the nonlinearity coordinates are considered for compression. It includes both the color model of CMYK and RGB. The 3D process is the $\mathrm{L}^{*} \mathrm{a} \mathrm{b}^{*}$ model to coordinate the color with lightness. The process of estimation of the salient feature (SF) is given below.

Read the input video

Split the video into frames

Convert the frames into LAB color space

Get the L,A,B layers from the LAB color space

Compute the mean for the each layer

Finally, calculate the salient feature by using the following formula.

$$
S F=\sqrt{\left(1-l_{m}\right)^{2}+\left(a-a_{m}\right)^{2}+\left(b-b_{m}\right)^{2}}
$$


Where, the layer of the color space are 1 , a and b. Here, $\mathrm{lm}$, am and bm are the values of the mean of color space layer respectively.

\subsection{Feature Extraction}

The frames of salient features including texture features are extracted. Here the contrast (I), mean (m), correlation (C), entropy (E), energy (En) and homogeneity (H) are estimated by using the following expression. The features are extracted based on the salient data.

$$
\begin{aligned}
& I(d, \theta)=\sum_{n, m}(n-m)^{2} C(d, \theta) \\
& m(\mathrm{~T})=\frac{1}{M \times N} \sum_{n=0}^{M-1} \sum_{m=0}^{N-1} T(n, m) \\
& C(d, \theta)=\frac{\sum_{n, m}\left(n-\mu_{x}\right)\left(m-\mu_{y}\right) C(d, \theta)}{\sigma_{x} \sigma_{y}} \\
& \mathrm{E}(d, \theta)=\sum_{n, m} C(d, \theta) \log C(d, \theta) \\
& E_{n}(d, \theta)=\sum_{n, m} c^{2}(d, \theta) \\
& H(d, \theta)=\sum_{n, m} \frac{1}{1+(n-m)^{2}} C(\mathrm{~d}, \theta)
\end{aligned}
$$

After the extraction of features, the redundancy of the data is removed by applying the similarity measurement which determines the similarity features and removes the redundancy from the data. The input texture data is compared with the extracted features by implementing the method of Euclidean distance.

$$
d_{s t}^{2}=\left(x_{s}-x_{t}\right)\left(x_{s}-x_{t}\right)^{\prime}
$$

Where $d_{s t}^{2}$ corresponds to similarity feature measure, where $x_{s}$ is input texture data and $x_{t}$ is extracted feature. According to the proposed model, if the features are same or similar then the corresponding input video frames will be treated as redundant information and are removed from the video. After the process of redundancy removal, the frames are split into background and foreground frames. Finally, both the frames are encoded by the enhanced run length coding scheme. The decoding process is carried out as encoding in the reverse process and is given below.

Set the previous symbol equal to an unmatchable value.

Read the next symbol from the input stream.

If the symbol is an EOF, then exit 
Else, obtain the current symbol.

If the symbol doesn't match the previous symbol, then set the previous symbol to the current symbol, and go to step b.

The additional symbol is counted and initialized until a non-matching symbol is found. This is the run length.

Obtain the run length outcomes and the non-matching symbol.

Set the previous symbol to the non-matching symbol, and go to step b.

\section{Performance Analysis}
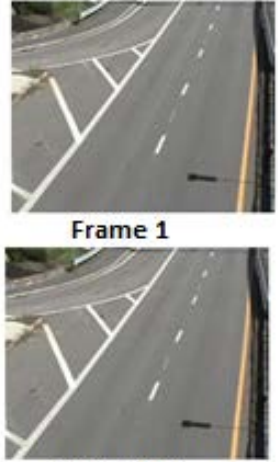

Frame 150

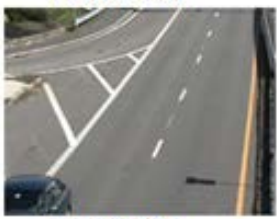

Frame 300

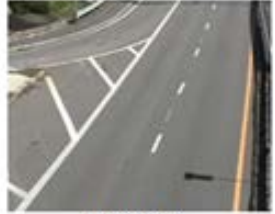

Frame 6

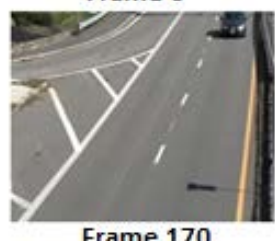

Frame 170

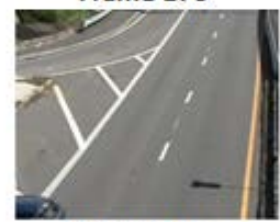

Frame 305

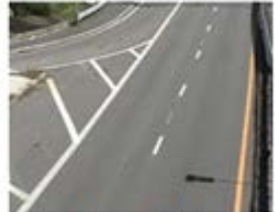

Frame 50

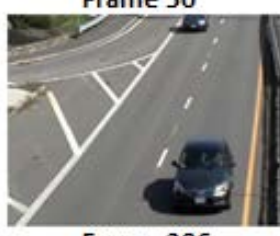

Frame 206

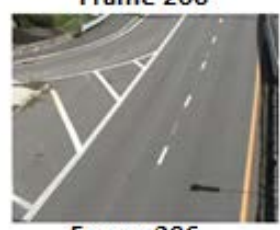

Frame 306

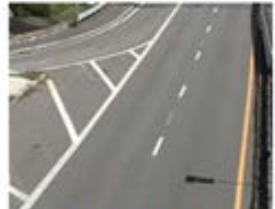

Frame 100

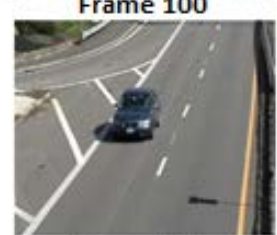

Frame 270

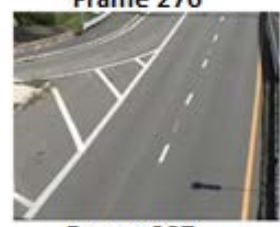

Frame 307

(a)

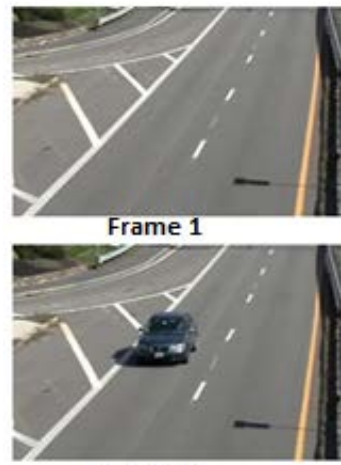

Frame 87

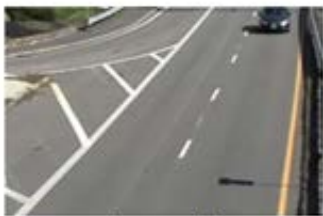

Frame 38

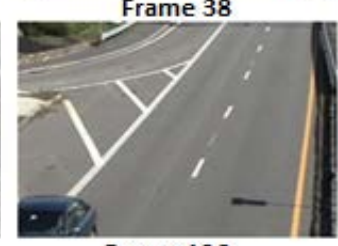

Frame 126

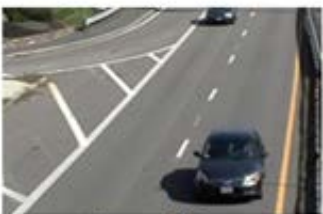

Frame 64

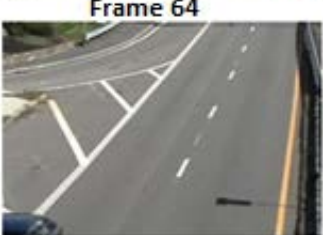

Frame 148

(b)

Figure 3. Input Frames

The performances of the proposed algorithm are simulated using MATLAB 8.2 
version software tool. A test surveillance video of size $3.12 \mathrm{Mb}$ (disk space) is taken to test the proposed algorithm. Initially, the test video is converted into frames and is shown in Figure 3 which comprises few test samples. The feature information is extracted from the input frames based on equation (2) to equation (7). Based on the feature information, the frames which are almost similar or having vast redundant information are eliminated. The remaining frames are clubbed together and are shown in Figure 4. From Figure 3, it is observed that frames 1 to frames 150 are having same or nearly equal information and hence are eliminated and shown in Figure 4.
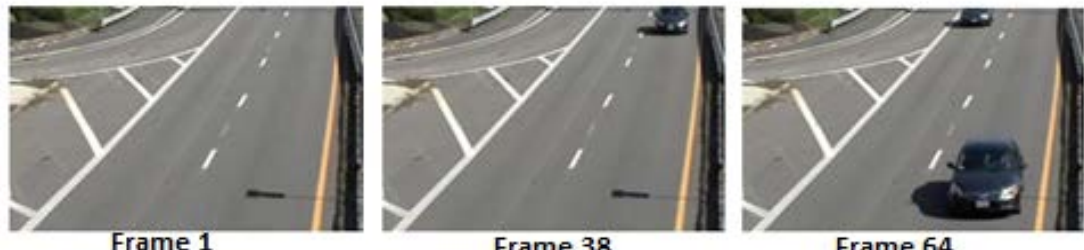

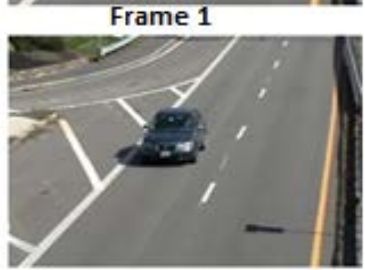

Frame 87

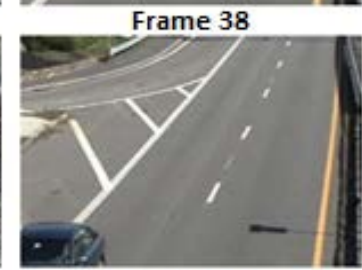

Frame 126

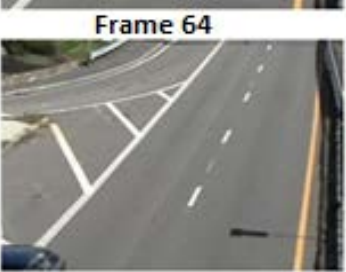

Frame 148

Figure 4. Encoded Frames (Redundancy Removal)

Decoding part of the proposed algorithm involves similar procedures in reverse order. Figure 5 shows the video output after decoding the compressed data having size $1.54 \mathrm{Mb}$ (disk space) which almost have the size of input data.
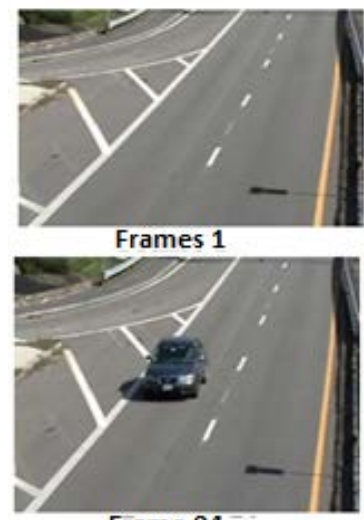

Frame 94

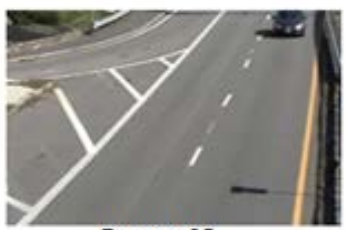

Frame 43

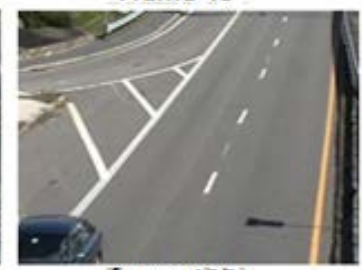

frame 135

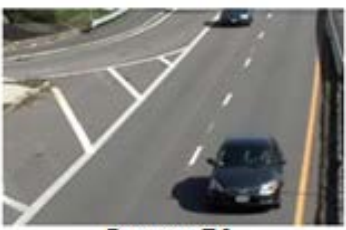

Frames 74

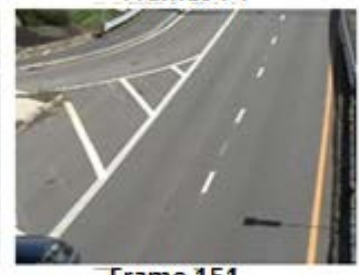

Frame 151

Figure 5. Decoded Frames

Figure 6 shows the comparison of average mean square error of the proposed model with existing methods like SPIHT\& Markov method. The average mean square error is computed by taking the average value of the mean square error of individual video input. It is observed that the proposed model performs better compared to other existing techniques. 


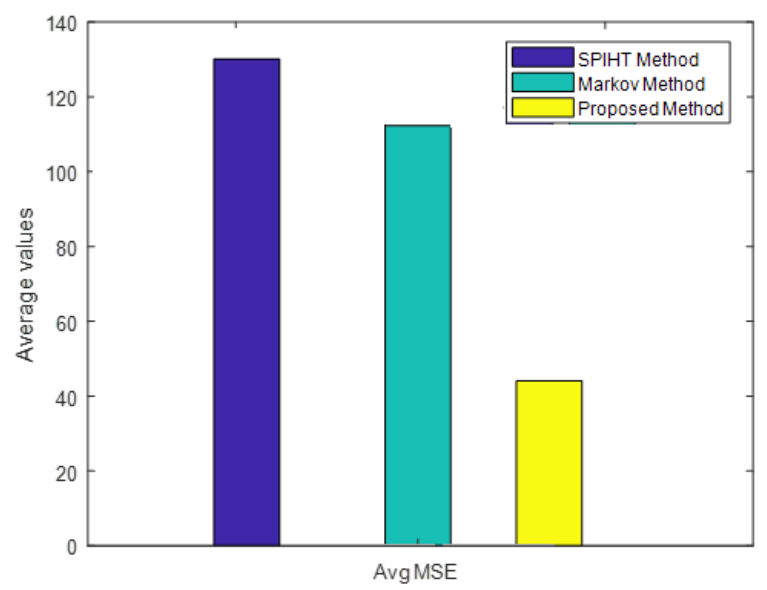

Figure 6. Average MSE values for proposed method

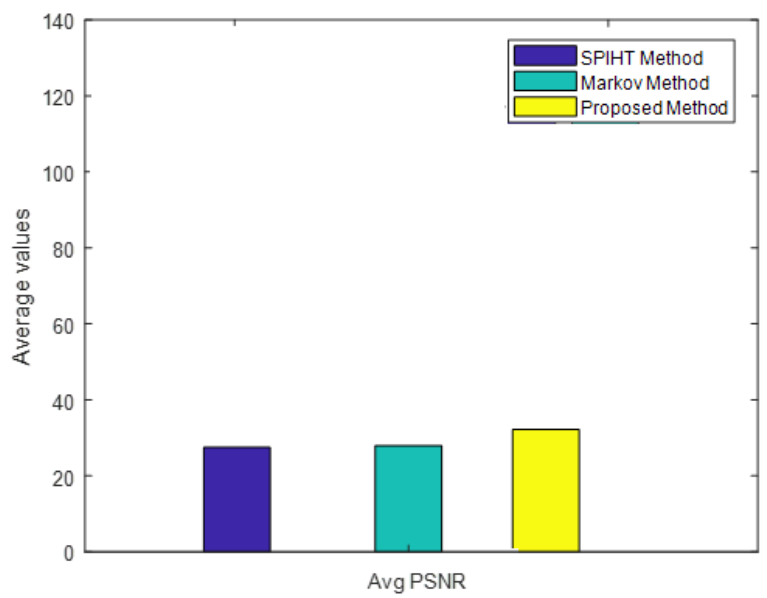

Figure 7. Average PSNR values for proposed method

Figure 7 shows the comparison of average peak signal to noise ratio of the proposed model with existing methods like SPIHT \& Markov method. The average peak signal to noise ratio is computed by taking the average value of peak signal to noise ratio of individual video input. It is observed that the proposed model performs better compared to other existing techniques. In order to have better compression rate, the proposed model compresses foreground and background information separately. This is due to the fact that the most background information of video sample input remained same when compared to the foreground data and hence the proposed model utilizes a lesser number of data bits to compress it. This results in a reduced number of bits required used in video compression when compared to other techniques. 


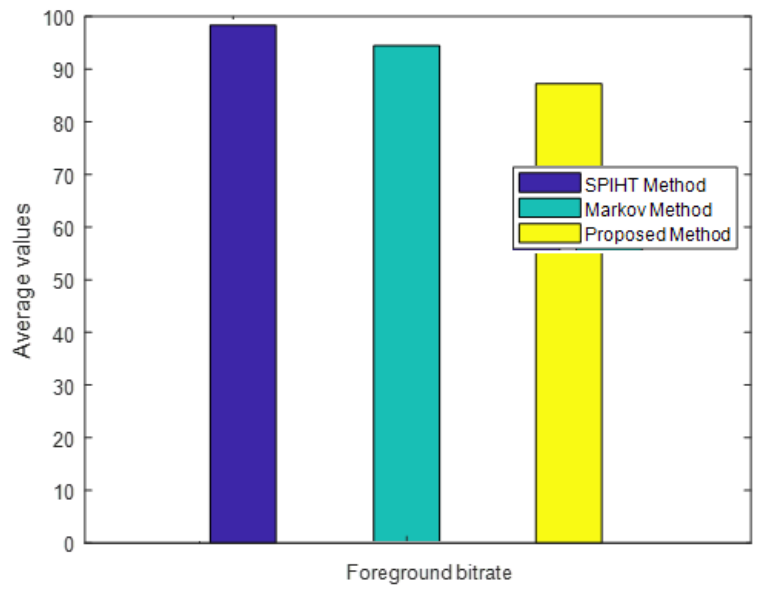

Figure 8. Average foreground bit rate values for proposed method

Figure 8 shows the comparison of foreground bitrate of the proposed model with existing methods like SPIHT \& Markov method. The average foreground bitrate is computed by taking the average value of foreground bitrate of individual video input. It is observed that the proposed model performs better compared to other existing techniques.Figure 9 shows the comparison of background bitrate of the proposed model with existing methods like SPIHT \& Markov method. The average background bitrate is computed by taking the average value of background bitrate of individual video input. It is observed that the proposed model performs better compared to other existing techniques.

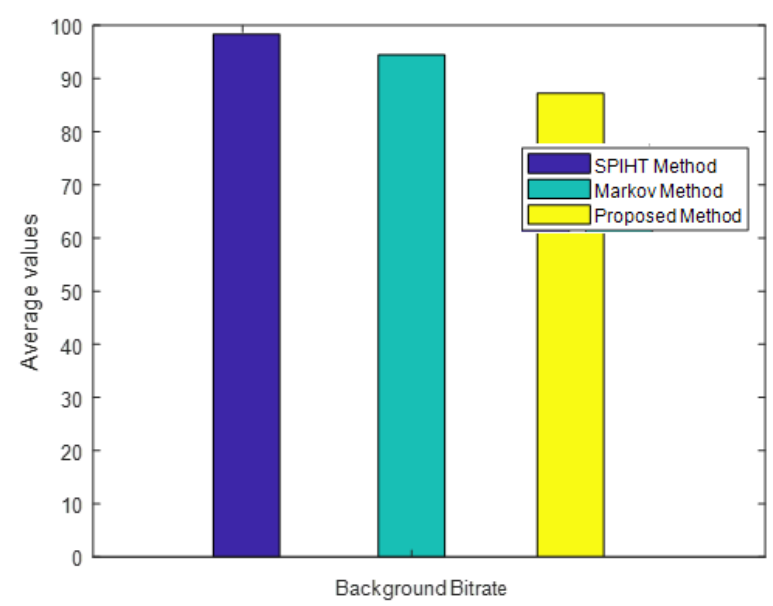

Figure 9. Average background bit rate values for proposed method

Figure 10 shows the comparison of the average execution time of the proposed model with existing methods like SPIHT \& Markov method. The average execution time is computed by taking the average value of execution time of individual sample video input. It is observed that the proposed model performs better compared to other existing techniques. The proposed model utilizes reduced number bits in compressing video inputs and hence the model achieves 50\% reduction in execution time when compared to SPIHT method and 15\% reduction in 
execution time when compared to Markov method

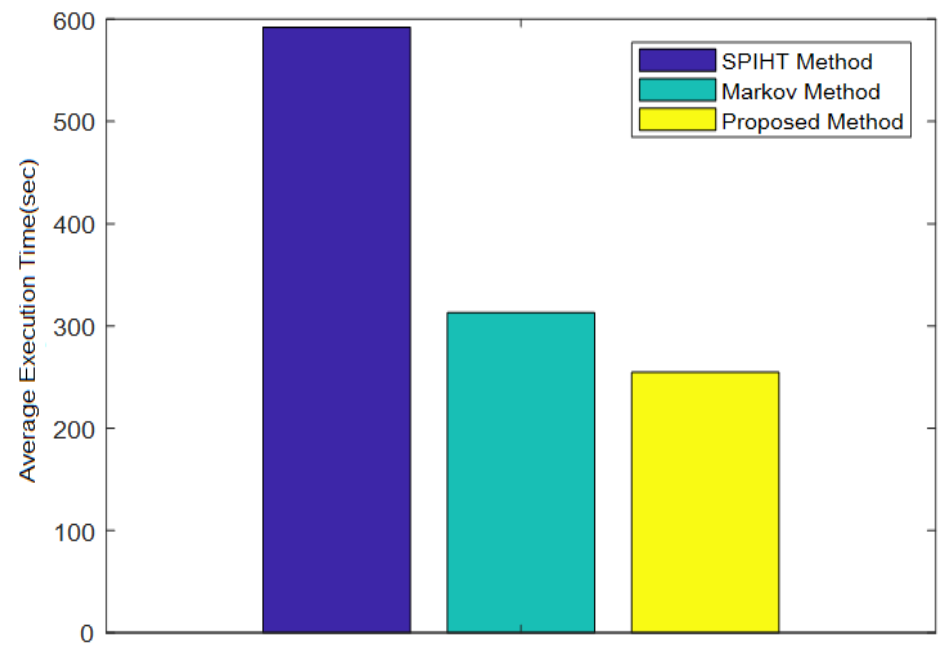

Figure 10. Average execution time values for proposed method

\section{Conclusion}

In this paper, a novel video compression algorithm is presented. The proposed algorithm involves extraction of feature information and thereby eliminating the redundant frames from the input video data. The algorithm achieves a compression rate of $50 \%$ when compared to the existing algorithm without much sacrificing the performance of the video information. The performance of the proposed method is validated using MATLAB and are compared with existing models including SPHIT and Markov's model, hence, it is proved that the proposed algorithm serves better in the big data surveillance system and real-time applications.

\section{References}

[1] Jing Xiao, Liang Liao, Jinhui Hu, Yu Chen, Ruimin Hu. 2015. Exploiting global redundancy in big surveillance video data for efficient coding. Springer Science Business Media New York, 18: pp. 531-540.

[2] Amritha, K. M. Nithin, S S. 2015. Adaptive Encoding \& Decoding of Compressed Video Using SPIHT Algorithm. International Journal of Advanced Research in Computer Engineering \& Technology (IJARCET), 4(5): pp. 2409-2412.

[3] Kamisli, F. 2015. Block-Based Spatial Prediction and Transforms Based on 2D Markov Processes for Image and Video Compression. IEEE Transactions on Image Processing, 24(4): pp.1247-1260.

[4] Ma, M. Hu, R. Chen, S. Xiao, J. Wang, Z. Qu, S. 2015. Global Object Representation of Scene Surveillance Video Based on Model and Feature Parameters. Advances in Multimedia Information Processing - PCM 2015, 9314: pp 223-232.

[5] Yin-Tsung, H. Ming-Wei, L. Cheng-Chen, L. 2015. A Low-Complexity Embedded Compression Codec Design With Rate Control for High-Definition Video. IEEE Transactions on Circuits and Systems for Video Technology, 
25(4): pp.674-687.

[6] Zhang, Y. Agrafiotis, D. Bull, D. R. 2013. High Dynamic Range image \& video compression a review. 2013 18th International Conference on Digital Signal Processing (DSP), Fira, pp. 1-7.

[7] Liu, F. Koenig, H. 2014. Puzzle-an efficient, compression independent video encryption algorithm. Multimedia Tools and Applications, 73(2): pp. 715-735.

[8] Xu, M. 2014. Compressibility constrained sparse representation with learnt dictionary for low bit-rate image compression. IEEE Trans. Circuits Syst. Video Technol,. 24(10): pp.1743-1757.

[9] Zhang, X. 2014. Background-modeling-based adaptive prediction for surveillance video coding. IEEE Trans. Image Process,. 23(2): pp. 769-784.

[10] AntoBennet, M. Nithyadevi, R. Jacob Reglend, I. Nagarajan, C. 2013. Performance and Analysis of Video Compression Using Block Based Singular Value Decomposition Algorithm. International Journal of Modern Engineering Research (IJMER), 3(3): pp-1482-1486.

[11] Chongyu Chen, JianfeiCai, Weisi Lin, Guangming Shi. 2012. Surveillance Video Coding via Low-Rank and Sparse Decomposition. Proceedings of the 20th ACM international conference on Multimedia (MM '1), pp. 713-716.

[12] Prakash, V.R. Nagarajan, S. 2019. Intelligent systems for redundancy removal with proficient run-length coding and statistical analysis using regression. International Journal of Intelligent Systems Technologies and Applications, 18(1): pp. 101-114.

[13] Prakash, V.R. Nagarajan, S. 2019. Utilizing global redundancy in BSVD using RR- ERLC for efficient video coding. International Journal of Control Theory and Applications, 9(34): pp. 833-841. 